\title{
BMJ Open Can traditional risk factors explain the higher risk of cardiovascular disease in South Asians compared to Europeans in Norway and New Zealand? Two cohort studies
}

\author{
Kjersti S Rabanal, ${ }^{1}$ Haakon E Meyer, ${ }^{1,2}$ Grethe S Tell, ${ }^{3,4}$ Jannicke Igland, ${ }^{4}$ \\ Romana Pylypchuk, ${ }^{5}$ Suneela Mehta, ${ }^{5}$ Bernadette Kumar, ${ }^{6}$ Anne Karen Jenum, ${ }^{7}$ \\ Randi M Selmer, ${ }^{1}$ Rod Jackson ${ }^{5}$
}

To cite: Rabanal KS, Meyer HE, Tell GS, et al. Can traditional risk factors explain the higher risk of cardiovascular disease in South Asians compared to Europeans in Norway and New Zealand? Two cohort studies. BMJ Open 2017;7:e016819. doi:10.1136/ bmjopen-2017-016819

- Prepublication history and additional material for this paper are available online. To view these files please visit the journal (http://dx.doi.org/10. 1136/bmjopen-2017-016819)

Received 13 March 2017 Accepted 8 August 2017

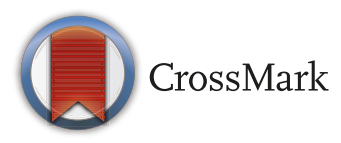

For numbered affiliations see end of article.

Correspondence to

Kjersti S Rabanal;

kjersti.stormark.rabanal@fhi.no

\section{ABSTRACT}

Objectives The objective was to prospectively examine potential differences in the risk of first cardiovascular disease (CVD) events between South Asians and Europeans living in Norway and New Zealand, and to investigate whether traditional risk factors could explain any differences.

Methods We included participants (30-74 years) without prior CVD in a Norwegian $(n=16606)$ and a New Zealand $(n=129449)$ cohort. Ethnicity and cardiovascular risk factor information was linked with hospital registry data and cause of death registries to identify subsequent CVD events. We used Cox proportional hazards regression to investigate the relationship between risk factors and subsequent CVD for South Asians and Europeans, and to calculate age-adjusted HRs for CVD in South Asians versus Europeans in the two cohorts separately. We sequentially added the major CVD risk factors (blood pressure, lipids, diabetes and smoking) to study their explanatory role in observed ethnic CVD risk differences.

Results South Asians had higher total cholesterol (TC)/ high-density lipoprotein (HDL) ratio and more diabetes at baseline than Europeans, but lower blood pressure and smoking levels. South Asians had increased age-adjusted risk of CVD compared with Europeans (87\%-92\% higher in the Norwegian cohort and $42 \%-75 \%$ higher in the New Zealand cohort) and remained with significantly increased risk after adjusting for all major CVD risk factors. Adjusted HRs for South Asians versus Europeans in the Norwegian cohort were $1.57(95 \% \mathrm{Cl} 1.19$ to 2.07$)$ in men and 1.76 $(95 \% \mathrm{Cl} 1.09$ to 2.82$)$ in women. Corresponding figures for the New Zealand cohort were 1.64 (95\% Cl 1.43 to 1.88) in men and $1.39(95 \% \mathrm{Cl} 1.11$ to 1.73$)$ in women. Conclusion Differences in TC/HDL ratio and diabetes appear to explain some of the excess risk of CVD in South Asians compared with Europeans. Preventing dyslipidaemia and diabetes in South Asians may therefore help reduce their excess risk of CVD.

\section{INTRODUCTION}

Immigrants from South Asia (countries in the Indian subcontinent, such as India, Pakistan,
Strengths and limitations of this study

- This is one of few prospective investigations of cardiovascular disease and its risk factors in South Asian populations living in Western countries.

- A special feature is the inclusion of prospective data from two different countries enhancing the external validity of the findings.

- The two cohorts differed in how participants were recruited and how information about risk factor levels was collected at baseline.

- A limited number of South Asians in the Norwegian cohort and short follow-up time in the New Zealand cohort restricted the statistical power in our analyses.

Sri Lanka and Bangladesh) who have settled in Western countries have increased risk of cardiovascular disease (CVD) compared with their host populations of European origin. ${ }^{1}$ This excess risk has been documented in several countries, especially the increased risk of coronary heart disease (CHD).$^{2-4} \mathrm{We}$ recently found that South Asian immigrants in Norway had more than twofold higher risk of acute myocardial infarction (AMI) than ethnic Norwegians and an increased risk of stroke (26\% higher in men and 58\% higher in women). ${ }^{5}$ Collaborators in New Zealand found a higher risk of CVD in Indians compared with the European New Zealand population. ${ }^{6}$

The mechanisms underlying the increased risk of CVD in South Asian populations are to a great extent unknown. ${ }^{1}$ Few studies have examined the prospective relationship between CVD risk factors and subsequent CVD among South Asians, ${ }^{4-9}$ despite the urgent need for such studies being addressed 
for more than 10 years ago. ${ }^{10}$ The two large and multinational case-control studies, INTERHEART ${ }^{11}$ and INTERSTROKE, ${ }^{12}$ indicate that different populations share the same risk factors and that the relationship between risk factors and CVD is similar in different populations around the world. The INTERHEART study also concluded that the earlier age of AMI in South Asians can be largely attributed to higher risk-factor levels at younger ages. ${ }^{13}$ However, the INTERHEART and INTERSTROKE studies are both case-control studies. In both Norway and New Zealand, South Asians have been found to have similar or higher mean total cholesterol (TC) to high-density lipoprotein (HDL) ratio and higher prevalence of diabetes compared with the European majority populations. ${ }^{14-17}$ However, they also have lower levels of smoking (especially women) and mean systolic blood pressure (SBP) than the European majority populations. Whether the higher risk of CVD among South Asians in Norway and New Zealand is due to higher levels of certain risk factors have not previously been studied.

Due to the dearth of prospective data on the relationship between risk factors and CVD among South Asians, we aimed to prospectively examine possible differences in the risk of a first CVD event between South Asians and Europeans using cohort studies from Norway and New Zealand, and to examine whether traditional CVD risk factors could explain such differences. Since the two cohorts differ in several aspects we do not intend to compare the two cohorts directly, but mainly focus on within-country comparisons.

\section{MATERIAL AND METHODS \\ The New Zealand PREDICT-CVD cohort}

We used data from the PREDICT-CVD cohort, collected through use of the PREDICT web-based decision support program in New Zealand for the assessment and management of CVD risk during primary healthcare consultations. ${ }^{18}$ The study methods and data definitions are described in detail elsewhere. ${ }^{18}{ }^{19}$ In short, the software has been integrated with commonly used primary care management systems, and allows systematically coded CVD risk data to be automatically and anonymously extracted from patients' electronic medical records and augmented where required by primary care staff. ${ }^{18} 19$ The cardiovascular profile data was subsequently linked, using an encrypted national health identifier number to national and regional health datasets with information about hospitalisations, deaths, publicly funded drug dispensing and laboratory test claims and results. ${ }^{19}$

The PREDICT software is used in around $35 \%$ of New Zealand primary care practices mainly in the Auckland and Northland regions, ${ }^{19}$ which serve around 1.7 million people, representing around $37 \%$ of the New Zealand population. ${ }^{20}$ Any patient with their CVD risk assessed by a general practitioner (GP) or practice nurse into online PREDICT-CVD forms are included in the PREDICT cohort.
New Zealand CVD risk management guidelines recommend that all men aged over 45 years and all women aged over 55 years have a regular CVD risk assessment. ${ }^{21}$ Specified high-CVD risk groups, including those of South Asian ethnicity, are recommended to undergo a risk assessment 10 years earlier than the general population.

We used PREDICT data from August 2002 to September 2012. Members of the cohort were enrolled and examined continuously throughout this period via their contact with the primary healthcare. We included individuals aged 30-74 years since the dataset comprised people undergoing a risk assessment based on a Framingham risk score intended for people in this age group. ${ }^{22}$ Using information from the GP, hospital discharges and medication dispensing, we excluded persons with a history of CVD (CHD (including angina), stroke, transient ischaemic attack (TIA), peripheral vascular disease, percutaneous coronary intervention or coronary artery bypass grafting), or atrial fibrillation at baseline $(\mathrm{n}=24537)$, and people with overt renal disease, those who had estimated glomerular filtration rate $\leq 29$ and those with prior hospitalisations for congestive heart failure (HF) or who were on loop diuretics at baseline $(n=1582)$. Only subjects with European or Indian background were included. The risk factor measurements in the PREDICT cohort were extracted from a standardised electronic template that primary care practitioners completed. The SBP was based on the mean of the last two recordings done by the GP or practice nurse, in most cases with a manual mercury sphygmomanometer. Blood lipid and glucose or glycated haemoglobin measurements were carried out in the community laboratories routinely used by GPs and smoking status and other risk factor data were measured using a standard questionnaire completed by a primary care practitioner.

\section{Cohort of Norway}

We included participants from three surveys conducted during 2000 to 2002 in Oslo, Norway; The Oslo Health Study (HUBRO), The Oslo Immigrant Health Study (I-HUBRO) and The Romsås in Motion study (MoRo II) $(\mathrm{n}=26$ 709), which are part of the Cohort of Norway $(\mathrm{CONOR})^{23}$; a collection of health data and blood samples from several Norwegian health surveys. Participation rates for the three studies were $40 \%-46 \%{ }^{23}$

All CONOR surveys followed the same standard procedure for collection of data from self-administered questionnaires, physical measurements and blood samples. The CONOR questionnaire provided information on self-reported diabetes, smoking, use of blood pressure (BP) and/or lipid-lowering medication and family history of CVD. All participants attended a clinical examination and non-fasting venous blood samples were drawn. SBP was measured by an automatic device (DINAMAP, Criticon, Tampa, FL, USA) after 2 min of seated resting. Three recordings were made at 1-min intervals. For the analyses we used the average of the second and third SBP measurements. The blood 
samples were subsequently measured for TC and HDL cholesterol. ${ }^{23}$

Using an 11-digit personal identifier, CONOR data were linked to hospitalisations and deaths in the Cardiovascular Disease in Norway (CVDNOR) project, 1994 to 2009. ${ }^{24} 25$ This enabled us to follow CONOR participants for CVD outcomes (hospitalisations or deaths) occurring after CONOR examination through 31 December 2009.

We included participants aged 30-74 years at baseline $(n=3871$ excluded) to ensure comparable samples between the Norwegian and New Zealand data. We excluded participants not born in Norway or South Asia ( $n=5651$ excluded), pregnant women $(n=197)$, and participants with prior CVD (CHD, cerebrovascular disease, atherosclerotic disease, TIA and HF) $(n=353)$ or atrial fibrillation $(n=31)$ registered in the hospital data before screening.

\section{Outcomes}

In both cohorts, we identified the first CVD event (non-fatal and fatal) using main or secondary diagnoses from hospital discharge data or the underlying cause of death from national mortality statistics. The International Classification of Diseases (ICD) codes (versions 9 and/or 10) were used to define outcome variables. New Zealand hospitals used an Australian modification of the ICD-10 classification called ICD10-AM. ${ }^{26}$

CVD in both cohorts included the following conditions: CHD; HF; cerebrovascular disease including TIA; diseases of arteries, arterioles and capillaries including atherosclerosis, aneurysm and dissection as well as embolism and thrombosis. For the Norwegian cohort, this included the codes: ICD9: 410-414, 428, 430-438, 440, 441 except 441.7, 442, 443.9, 444; ICD10: I20-I25, I50, I60-I69, I70I79, G45. The CVD variable in the New Zealand PREDICT cohort included the same ICD10 codes as just listed, and also some additional ICD10-codes (I469, J81, G460G468, Z951, Z955, Z958, Z959) plus a list of procedure codes (too many to be listed here). The PREDICT-CVD outcome has been described elsewhere. ${ }^{19}$

\section{Ethnicity}

Ethnicity in the New Zealand PREDICT data was based on two sources: (1) the PREDICT template filled in by the GP and (2) the National Health Index dataset, both according to pre-defined categories. A prioritising algorithm was used to agree on one ethnicity in case of multiple ethnicities recorded (details can be found in the online supplementary file entitled the VIEW Ethnicity Protocol). The system for coding ethnicity in New Zealand enables identification of Indian people, who account for approximately $90 \%$ of South Asian people living in New Zealand. The remaining South Asian ethnic groups are classified as part of the 'Other Asian' ethnic group in national health data and so could not be included here. Indian people can include both immigrants and individuals who have been born in New Zealand with parents (or older generations) who have immigrated. The majority of this group are immigrants since $76.5 \%$ of the people who identified themselves with the Indian ethnic group in New Zealand in 2013 were born overseas. ${ }^{27}$

For the Norwegian cohort, we used country of birth merged into larger world regions to define ethnicity. ${ }^{28}$ We defined South Asians as individuals who migrated to Norway from Bangladesh, Myanmar, Sri Lanka, Pakistan, India or Nepal. ${ }^{28}$ The largest share of South Asians in this dataset $(95 \%)$ came from the HUBRO or the I-HUBRO study. HUBRO and I-HUBRO combined included 1145 Sri Lankans and 780 Pakistanis, ${ }^{29}$ indicating that about $50 \%$ of the South Asian group $(\mathrm{n}=2206)$ in the present study are Sri Lankans and 35\% are Pakistanis.

In general, we refer to the ethnic groups as South Asians (South Asians in Norway and/or Indians in New Zealand) and Europeans (ethnic Norwegians and/or New Zealanders with ethnic European origin). Most European New Zealanders are of British and Irish ancestry, of whom about three-quarters were born in New Zealand.

\section{Statistical analysis}

Baseline characteristics are reported as mean values with SD for continuous variables and fractions for categorical variables. We tested the differences between the ethnic groups adjusted for age by analysis of covariance. We used Cox regression models to examine the prospective relationship between baseline risk factors (BP, lipids, diabetes and smoking) and time until subsequent first CVD event. People were censored if they died from other causes ( $\mathrm{n}=961$ in PREDICT and $\mathrm{n}=276$ in CONOR). Cox regression was also used to calculate HRs for CVD in South Asians versus Europeans using ethnicity as the exposure variable and adjusting for risk factors. The order we added the risk factors to the model was based on the distribution of risk factors in the subpopulations. This meant that we first introduced the risk factors that were more prevalent among South Asians compared with Europeans (diabetes and TC/HDL ratio) and then added the two less prevalent risk factors (SBP and smoking). Additional analyses where we added the risk factors in different orders and looked at each risk factor in separate models with only age as covariate did not change the conclusions (Tables A1 and A2 in the online supplementary appendix). Proportional hazards assumptions were tested using scaled Schoenfeld residuals. ${ }^{30}$ All analyses were stratified by sex and ethnicity, except for the analyses where ethnicity was the exposure variable in which we only stratified by sex. Only complete cases were included in the analyses. Stata V.14 was used for analyses in the Norwegian data and Stata V.11 for analysis in the New Zealand data.

To check whether the use of BP medication at baseline would impact the analyses where SBP were included, we repeated the Cox regression analyses excluding people using antihypertensive medication at baseline. Correspondingly, we also repeated the Cox regression analyses for TC/HDL ratio without people using lipid-lowering medication at baseline. In addition, since excluding those 
at highest risk could potentially impact the sensitivity analyses, we also adjusted for medication use without excluding anyone from the analyses (Tables A3and A4 in the online supplementary appendix).

\section{Ethics}

The current project was approved by the Regional Committee for Medical Research Ethics, Health Region West. The PREDICT study was approved by the Northern Region Ethics Committee Y in 2003 (AKY/03/12/134), and later annually approved by the National Multi Region Ethics Committee since 2007 (MEC07/19/EXP). ${ }^{19}$ Each individual CONOR study was approved by the Norwegian Data Inspectorate and evaluated by the Regional Committee for Medical Research Ethics. ${ }^{31}$ Both datasets contained anonymised data.

\section{RESULTS}

\section{Baseline characteristics}

The final study sample from the New Zealand cohort consisted of 129449 individuals (43\% women) of European $(87 \%)$ or Indian ethnicity $(13 \%)$ with no history of CVD, atrial fibrillation or renal disease. Correspondingly for the Norwegian cohort, the final study sample consisted of 16606 individuals (54\% women) born in either Norway $(87 \%)$ or South Asia $(13 \%)$ with no history of CVD or atrial fibrillation.

At baseline, the Norwegian cohort was younger than the New Zealand cohort, and New Zealand women were older than New Zealand men (table 1). In both cohorts, South Asians were younger than Europeans.

South Asians had lower levels of TC and HDL and higher mean levels of TC/HDL ratios than Europeans in both Norway and New Zealand. South Asians also had the lowest SBP levels (table 1). These differences persisted after adjustment for age $(\mathrm{P}<0.05$ for differences between ethnic groups-results not shown).

The diabetes baseline prevalence was higher among South Asians compared with Europeans in both cohorts (table 1). The difference in diabetes were the same after adjustment for age $(\mathrm{P}<0.001)$. Antihypertensive and lipid-lowering treatments were generally more prevalent among South Asians than Europeans, and more prevalent in the New Zealand cohort compared with the Norwegian cohort. Cigarette smoking was more common among Europeans than South Asians, and practically none of the South Asian women smoked. Mean follow-up time was significantly longer in the Norwegian cohort than in the New Zealand cohort (table 1).

\section{CVD events}

During follow-up, we observed 2654 CVD events among 129449 individuals in the New Zealand cohort (378 874 person-years) and 743 new CVD events among the 16606 individuals in the Norwegian cohort (139 470 personyears). The overall crude rates were 700 per 100000 person-years in the New Zealand cohort and 533 per 100
000 person-years in the Norwegian cohort. Ethnic specific rates for men and women in the two cohorts are shown in table 2 and in Tables A5-A8 of the online supplementary appendix. Also crude rates and age-adjusted HRs of CVD by risk factors, ethnic groups, cohort and gender can be found in the same tables (online supplementary appendix).

\section{Prospective associations between risk factors and CVD}

Increasing age was significantly associated with risk of CVD in both ethnic groups in both cohorts (table 2). The age effect was very similar within the countries for both ethnic groups and gender, but was stronger in the Norwegian cohort compared with the New Zealand cohort. After adjustment for age, the traditional CVD risk factors were positively associated with CVD in both ethnic groups, across gender and country. Whereas all the risk factor-CVD event associations were statistically significant in Europeans, the 95\% CIs were wider and the results not always statistically significant among South Asians. The relationship between SBP, TC/HDL ratio, smoking and subsequent CVD appeared to be weaker in Indian men compared with European men in the New Zealand cohort. The prospective association between the risk factors and CVD changed little after adjusting for the other risk factors in addition to age (results not shown). In the sensitivity analyses where we either adjusted for medication use (Table A3 in the Appendices) or excluded people using BP- and lipid lowering medication at baseline (results not shown), the estimates for the prospective associations between risk factors and CVD were similar as in the main analyses. However, for women in the New Zealand cohort, after excluding people on lipid-lowering medication, the HR for TC/HDL ratio changed to 1.12 (95\% CI 0.91 to 1.39 ) for Indian women and to 1.20 (95\% CI 1.12 to 1.27) for European women.

\section{Ethnic difference in CVD}

South Asians of both genders in Norway and New Zealand had increased risk of CVD compared with the European majority populations (table 3), with age-adjusted HRs ranging from 1.42 to 1.92. After adjustment for TC/HDL ratio and diabetes, the HRs for South Asians versus Europeans were reduced and no longer significant in women. Additional adjustments for SBP and smoking increased the HRs again so that South Asians in both countries had significantly increased risk of CVD compared with Europeans. After adjustment for age, TC/HDL ratio, diabetes, SBP and smoking, the HRs for the excess risk in South Asians compared with Europeans varied from 1.39 to 1.76. The largest reduction in risk estimate after full adjustment was seen in South Asian men in the Norwegian cohort where the HR was lowered by approximately $38 \%$ after adjusting for the four major risk factors. The smallest reduction in risk estimate after adjustment was among South Asian women in the New Zealand cohort where the risk estimate was only reduced by $7 \%$ (from 1.42 to 1.39 ). 
Table 1 Baseline characteristics (unadjusted) of the Norwegian and New Zealand participants. Participants free of prior CVD.

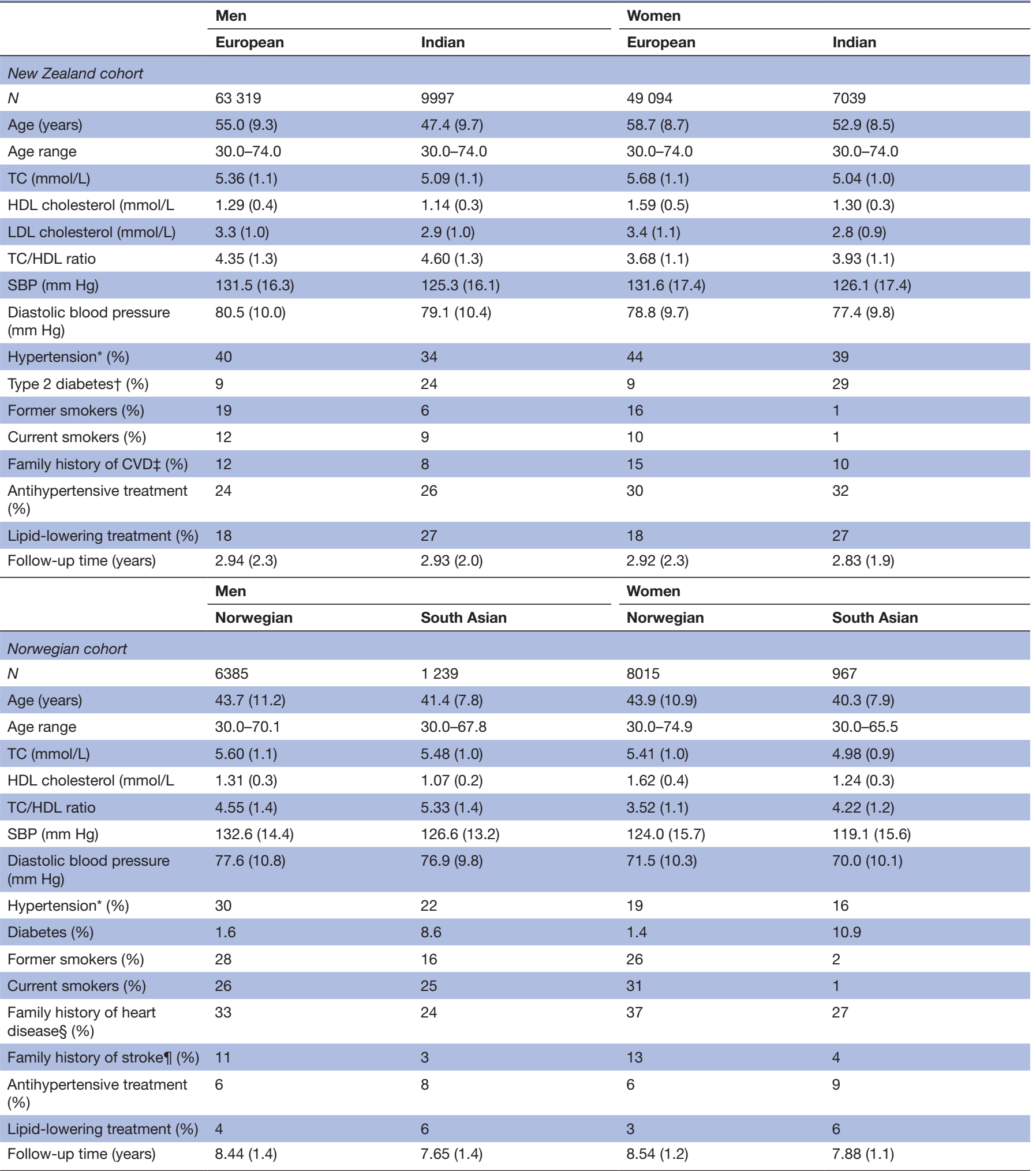

*Hypertension is defined as having SBP $\geq 140 \mathrm{~mm} \mathrm{Hg}$ or diastolic blood pressure $\geq 90 \mathrm{~mm} \mathrm{Hg}$ or using blood pressure medication.

†The diabetes variable in the New Zealand data includes people with diabetes of unknown type (5\%) and type 2 diabetes (95\%), while in the Norwegian data we could not differentiate between different types of diabetes.

†Family history of CVD in the New Zealand data: self-reported familial history of ischaemic heart disease or ischaemic stroke occurring in a father or brother aged $<55$ years, or a mother or sister aged $<65$ years.

§Parents or siblings have had heart attack or angina pectoris (self-report).

ПParents or siblings have had stroke (self-report).

Data are mean values (SD) for continuous variables and prevalence (\%) for categorical variables.

CVD, cardiovascular disease; HDL, high-density lipoprotein; LDL, low-density lipoprotein; SBP, systolic blood pressure, TC, total cholesterol. 


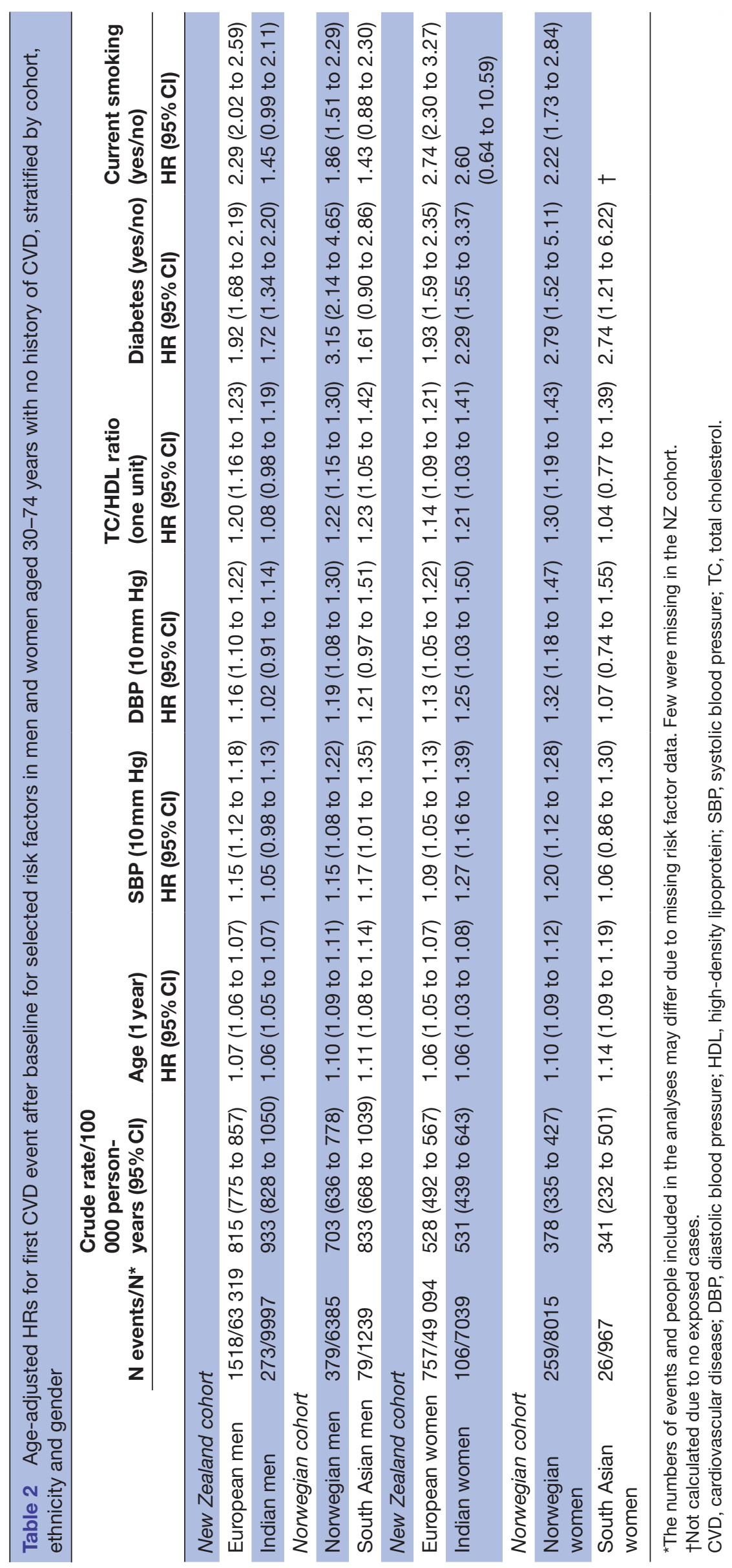


Table 3 HRs $(95 \% \mathrm{Cl})$ for first CVD event in South Asian groups compared with ethnic European groups in New Zealand and Norway

\begin{tabular}{|c|c|c|c|c|}
\hline & \multicolumn{2}{|l|}{ Men } & \multicolumn{2}{|l|}{ Women } \\
\hline & $\begin{array}{l}\text { Indian NZ versus } \\
\text { European NZ }\end{array}$ & $\begin{array}{l}\text { South Asians } \\
\text { versus Norwegians }\end{array}$ & $\begin{array}{l}\text { Indian NZ versus } \\
\text { European NZ }\end{array}$ & $\begin{array}{l}\text { South Asians } \\
\text { versus } \\
\text { Norwegians }\end{array}$ \\
\hline $\mathrm{N}$ events/N & $1791 / 73308$ & $436 / 7387$ & $863 / 56126$ & $264 / 8558$ \\
\hline \multicolumn{5}{|l|}{ Adjusted for } \\
\hline Age & 1.75 (1.53 to 2.00$)$ & $1.92(1.48$ to 2.49$)$ & $1.42(1.16$ to 1.75$)$ & $1.87(1.21$ to 2.87$)$ \\
\hline Age, TC/HDL ratio, diabetes & $1.49(1.30$ to 1.71$)$ & $1.42(1.08$ to 1.87$)$ & 1.15 (0.92 to 1.42$)$ & $1.30(0.82$ to 2.04$)$ \\
\hline Age, TC/HDL ratio, diabetes, SBP & $1.57(1.37$ to 1.80$)$ & $1.53(1.16$ to 2.01$)$ & $1.19(0.96$ to 1.47$)$ & $1.31(0.83$ to 2.07$)$ \\
\hline Age, TC/HDL ratio, diabetes, SBP, smoking & $1.64(1.43$ to 1.88$)$ & 1.57 (1.19 to 2.07$)$ & 1.39 (1.11 to 1.73$)$ & $1.76(1.09$ to 2.82$)$ \\
\hline
\end{tabular}

All had complete information on the risk factors.

CVD, cardiovascular disease; HDL, high-density lipoprotein; NZ, New Zealand; SBP, systolic blood pressure; TC, Total cholesterol.

Additional analyses showed that the excess risk in South Asians was particularly high for CHD. The full-adjusted HRs for CHD (corresponding to the analyses in the last row of table 3) were 2.07 (95\% CI 1.76 to 2.44 ) in South Asian men and 1.60 (95\% CI 1.20 to 2.13) in South Asian women in New Zealand. In the Norwegian cohort, the full-adjusted HRs for CHD were 1.86 (95\% CI 1.36 to 2.55) in South Asian men and 2.84 (95\% CI 1.61 to 5.03) in South Asian women (Table A9 in the Appendices). In the sensitivity analyses for table 3 where we excluded people using BP-lowering or lipid-lowering medication at baseline (results not shown) or adjusted for BP-lowering or lipid-lowering medication (Table A4 in the Appendices), the patterns according to the risk factor adjustments remained the same as in the main analysis.

\section{DISCUSSION}

This study confirmed that the traditional risk factors SBP, TC/HDL ratio, diabetes and smoking are all positively associated with risk of CVD in South Asians as well as in Europeans. The present study also confirmed that South Asians had an increased risk of CVD compared with Europeans and that ethnic differences in the distribution of TC/HDL ratio and type 2 diabetes appear to explain some of this excess risk.

The main strengths of this study are the prospective study design, and inclusion of data from two countries. Unfortunately, we lacked information about duration of stay for the immigrants, and the ethnic groups that we studied are heterogeneous.

Strengths of the PREDICT cohort are the large sample size and the completeness of risk factors included in the risk-assessment. Only $0.01 \%$ were missing on any of the four major risk factors because they were part of the prediction algorithm and thereby compulsory to fill in to the PREDICT template. Furthermore, comprehensive national health registers were used to identify and exclude people with prior CVD and to determine cardiovascular outcomes. In the New Zealand cohort, some recruitment bias is likely since risk assessment was initially prioritised for high-risk patients. Indian patients are therefore over-represented in the cohort together with Maoris and Pacifics. ${ }^{19}$ The representativeness of the source population is, however, improving as PREDICTs coverage increases. In this study, follow-up extended to 2012 when PREDICT included 50\% of guideline-eligible patients in the practices where the PREDICT software is used. ${ }^{19}$ We did not assume that the cohorts were representative of the general populations in the two countries, but that the ethnic groups within the two cohorts should be comparable. Adjusting for age was therefore particularly important in the New Zealand cohort since South Asians were around 7 years younger than Europeans. Results from the two cohorts showed approximately the same regarding ethnic differences, which is a strength concerning the external validity of these results. A limitation in the New Zealand data is short follow-up time restricting the statistical power. Another limitation is the lack of standardised BP measurements since recorded BP can easily be affected by a range of factors including the type of device used. ${ }^{32}$

Strengths of CONOR data are the standardised measurements of risk factors, the linkage with disease outcomes from comprehensive national health registers and the standardised way of defining ethnicity using country of birth. A validation study examining the Oslo Health study, showed that participants with a non-Western background had a lower participation rate than others. ${ }^{33}$ This may reflect self-selection, which can work both ways; healthy and resourceful people have the energy and motivation to participate or less healthy people who think their health could benefit from participating do so. Self-selection is unlikely to influence associations between risk factors and subsequent disease, but could influence the ethnic comparisons if the mechanisms were systematically different for the ethnic groups. The South Asian 
group in the Norwegian cohort was relatively small, which reduced the precision of the estimates and limited the statistical power. Another limitation in the CONOR data is missing information on some of the risk factors (see Tables A5-A8 in the Appendices for numbers of missing). However, the extent of missing was small. The risk factor with most missing in CONOR was diabetes (3\% for the total cohort).

In both cohorts, the endpoints are based on register data, including both hospital and mortality data, which enables almost complete ascertainment of CVD events. In New Zealand, more than $95 \%$ of patients with an acute CVD event are managed by government-funded health services. ${ }^{19}$ However, CVD events occurring among participants who travelled outside of New Zealand, those who emigrated after the index CVD risk assessment or among participants treated in private hospitals would not be captured in the national hospital and mortality registers. ${ }^{19}$ We have no information about possible emigration for the New Zealand cohort, but for the Norwegian cohort we know that few people have emigrated (about $1 \%$ of the ethnic Norwegians and $<3 \%$ of the South Asians who participated in the Oslo health studies had emigrated by the end of follow-up). A limitation for both cohorts is also the lack of medication data during follow-up. However, adjustment for baseline medication did not change the estimates (Tables A3-A4 in the Appendices), and table 1 shows that South Asians used more antihypertensives and lipid-lowering drugs at baseline than Europeans. Both countries have universal healthcare and South Asians should have the same access to cardiovascular medication as Europeans. It is therefore not likely that lack of treatment explains the differences in risk of CVD between the two ethnic groups.

Our finding that the traditional major CVD risk factors contribute to the development of CVD in South Asians as in Europeans was an expected, yet important, finding since most knowledge about CVD prevention is based on studies in populations of European descent, and some have questioned whether these risk factors apply worldwide. ${ }^{11} 34$ This finding is in line with the large INTERHEART and INTERSTROKE case-control studies, ${ }^{11} 12$ which reported that $90 \%$ of the population attributable risk for AMI and stroke worldwide was accounted for by, respectively, nine and ten (similar) risk factors, including those included in the present study. We are only aware of two other prospective studies reporting HRs for the prospective relationship between major CVD risk factors and subsequent CVD in South Asians. ${ }^{73}$ One of these studies included only men, ${ }^{7}$ and the other showed estimates for men and women combined and did not include blood lipids. ${ }^{35}$ These studies generally agree with our findings that traditional risk factors contribute to the development of CVD in South Asians as in Europeans. ${ }^{735}$ Also, consistent with previous reports, ${ }^{56}$ we found that South Asians in both Norway and New Zealand have a higher risk of CVD compared with the European majority populations. By including all the measured risk factors
(BP, TC/HDL ratio, diabetes and smoking) as adjustment variables in one statistical model, we could not explain the higher risk of CVD in South Asians. However, the increased risk was attenuated when we only included the risk factors more prevalent in South Asians than in Europeans (TC/HDL ratio and diabetes).

The excess risk of CVD among South Asians compared with Europeans in the Norwegian cohort was almost two-fold. This is comparable to what we reported previously when studying the total Norwegian population. ${ }^{5}$ The South Asians in the New Zealand cohort had $42 \%-75 \%$ higher risk of CVD compared with European New Zealanders, which also agrees with previous New Zealand studies. ${ }^{6}$ In both the Norwegian and New Zealand data, South Asians had higher baseline levels of dyslipidaemia indicated by the TC/HDL ratio and higher diabetes prevalence compared with the European majority populations, which is in general agreement with previous knowledge from these countries. ${ }^{14-16}$ Attenuation of the excess risk in South Asians versus Europeans was best achieved in the Cox model only including diabetes and TC/HDL ratio as covariates in addition to age. The same was found in both cohorts, clearly indicating that the unfavourable distribution of blood lipids and type 2 diabetes explains some of the higher risk of CVD in South Asians. South Asians generally have a high prevalence of metabolic risk factors related to insulin resistance, often clustered so that they match the concept of the metabolic syndrome. ${ }^{36-39}$ A British cohort study that tested whether traditional risk factors could account for the high mortality of CHD among South Asian men compared with European men, reported that adjusting for insulin resistance, dyslipidaemia and hyperglycaemia in South Asians did not explain their higher risk. ${ }^{7}$ However, they also adjusted for smoking and TC, which were both less prevalent/lower among South Asian men compared with European men.

It is unclear why the traditional risk factors do not completely explain the excess risk of CVD in South Asians. This could be related to incomplete adjustments; due to either imprecise measurement of risk factors or that other important risk factors were not included (eg, waist measurement, length of time since diabetes diagnosis). A number of non-conventional risk factors are also thought to partially account for the high risk of CVD in South Asians, including dysfunctional HDL, C reactive protein, thrombogenic risk factors, telomere length, high homocysteine levels and low birth weight. ${ }^{40} 41$ Socioeconomic factors could probably also explain some of the differences in risk between the ethnic groups, but we did not have such variables. Another possibility is that risk factors work cumulatively over time in the development of atherosclerosis, and some risk factors may also work at specific and crucial time points during the life course. Measurements taken on single occasions may also lead to an underestimation of the strength between the usual levels of the risk factors and later disease, known as the regression dilution bias. ${ }^{42}$ Consequently, it is unlikely 
that the ethnic differences would disappear completely by adjusting for selected risk factors measured once in midlife.

Although South Asians seem to have an underlying susceptibility for metabolic diseases, traditional and modifiable risk factors are important for preventing disease. Our analyses indicate that it is important to focus on the prevention of type 2 diabetes and dyslipidaemia when aiming to reduce the burden of CVD among South Asians. The additional effect of abdominal obesity for the risk of CVD among South Asians in Norway and New Zealand has, however, not yet been studied although we know that the prevalence is high in this ethnic group. ${ }^{37}{ }^{43} \mathrm{In}$ both Norway ${ }^{44}$ and New Zealand, ${ }^{46}$ intervention studies targeting immigrants from South Asia have been carried out with some promising results. A UK study that prospectively examined the influence from four health behaviours on the risk of CVD in South Asian immigrants and UK Europeans found an important potential for disease prevention among South Asians if they adhered to healthy behaviours. ${ }^{8}$

\section{CONCLUSION}

Ethnic differences in distribution of TC/HDL ratio and type 2 diabetes explained some, but not all, of the excess risks of CVD in South Asians compared with Europeans in Norway and New Zealand. Smoking and elevated BP were less prevalent among South Asians and thus could not explain any of the observed differences in risk of CVD. Targeted diabetes and dyslipidaemia management among South Asians, including support for healthy lifestyle choices, should be a priority if the high burden of CVD in these ethnic populations is to be reduced.
Author affiliations
${ }^{1}$ Division for Mental and Physical Health, Norwegian Institute of Public Health, Oslo, Norway
${ }^{2}$ Department of Community Medicine, Institute of Health and Society, University of Oslo, Oslo, Norway
${ }^{3}$ Division for Health Data and Digitalisation, Norwegian Institute of Public Health, Bergen, Norway
${ }^{4}$ Department of Global Public Health and Primary Care, University of Bergen, Norway ${ }^{5}$ School of Population Health, University of Auckland, Auckland, New Zealand ${ }^{6}$ Norwegian Center for Minority Health Research, Oslo, Norway ${ }^{7}$ Faculty of Health and Society, Institute of Health and Society, University of Oslo, Oslo, Norway

\begin{abstract}
Acknowledgements The authors thank Tomislav Dimoski at the Norwegian Knowledge Centre for Health Services, 0slo, Norway, for his contribution by developing a software necessary for obtaining data from Norwegian hospitals, conducting the data collection and quality assurance of data in this project. We also wish to thank Dr Geeta Gala and Dr Roshini Peiris-John, members of the South Asian governance group for the University of Auckland VIEW research team, for reviewing the paper and giving us their feedback.

Contributors HEM, RJ and GST contributed to the conception and design of the work. RJ, BK, AKJ and GST contributed to the collection of data. JI, RP and SM contributed to data preparations and definition of endpoints. KSR drafted the paper and carried out the data analyses. All authors contributed to the interpretation of data as well as critical reading and revision of the draft.
\end{abstract}

Funding This work was supported by the Norwegian Extra-Foundation for Health and Rehabilitation (grant number 2012-2-0129).
Competing interests All authors have completed the ICMJE uniform disclosure form at www.icmje.org/coi_disclosure.pdf. RJ and SM report grants from Health Research Council of New Zealand. All other coauthors have no competing interests to declare.

Ethics approval For the Norwegian cohort: the Regional Committee for Medical Research Ethics, Health Region West, Norway. For the New Zealand cohort: the Northern Region Ethics Committee $Y$ in 2003, and later annually approved by the National Multi Region Ethics Committee since 2007.

Provenance and peer review Not commissioned; externally peer reviewed. Data sharing statement № additional data are available.

Open Access This is an Open Access article distributed in accordance with the Creative Commons Attribution Non Commercial (CC BY-NC 4.0) license, which permits others to distribute, remix, adapt, build upon this work non-commercially, and license their derivative works on different terms, provided the original work is properly cited and the use is non-commercial. See: http://creativecommons.org/ licenses/by-nc/4.0/

(c) Article author(s) (or their employer(s) unless otherwise stated in the text of the article) 2017. All rights reserved. No commercial use is permitted unless otherwise expressly granted.

\section{REFERENCES}

1. Zaman MJ, Bhopal RS. New answers to three questions on the epidemic of coronary mortality in south Asians: incidence or case fatality? Biology or environment? Will the next generation be affected? Heart 2013;99:154-8.

2. Bo A, Zinckernagel L, Krasnik A, et al. Coronary heart disease incidence among non-Western immigrants compared to Danish-born people: effect of country of birth, migrant status, and income. Eur $J$ Prev Cardiol 2015;22:1281-9.

3. Hedlund E, Lange A, Hammar N. Acute myocardial infarction incidence in immigrants to Sweden. Country of birth, time since immigration, and time trends over 20 years. Eur J Epidemiol 2007;22:493-503.

4. Tillin T, Hughes AD, Mayet J, et al. The relationship between metabolic risk factors and incident cardiovascular disease in Europeans, South Asians, and African Caribbeans: SABRE (Southall and Brent Revisited) -- a prospective population-based study. J Am Coll Cardiol 2013;61:1777-86.

5. Rabanal KS, Selmer RM, Igland J, et al. Ethnic inequalities in acute myocardial infarction and stroke rates in Norway 19942009: a nationwide cohort study (CVDNOR). BMC Public Health 2015;15:1073.

6. Ministry of Health. Asian Health Chart Book 2006. Wellington: Ministry of Health, 2006.

7. Forouhi NG, Sattar N, Tillin T, et al. Do known risk factors explain the higher coronary heart disease mortality in South Asian compared with European men? Prospective follow-up of the Southall and Brent studies, UK. Diabetologia 2006;49:2580-8.

8. Eriksen A, Tillin T, O'Connor L, et al. The impact of health behaviours on incident cardiovascular disease in Europeans and South Asians-a prospective analysis in the UK SABRE study. PloS one 2015;1:15.

9. de Munter JS, Agyemang C, Stronks K, et al. Association of physical activity, smoking, and alcohol intake with CVD-related hospital discharge in people of European, South Asian, or African descent. Eur J Prev Cardiol 2013;20:80-8.

10. Ranganathan M, Bhopal R. Exclusion and inclusion of nonwhite ethnic minority groups in 72 North American and European cardiovascular cohort studies. PLoS Med 2006;3:e44.

11. Yusuf S, Hawken S, Ounpuu S, et al. Effect of potentially modifiable risk factors associated with myocardial infarction in 52 countries (the INTERHEART study): case-control study. Lancet 2004;364:937-52.

12. O'Donnell MJ, Xavier D, Liu L, et al. Risk factors for ischaemic and intracerebral haemorrhagic stroke in 22 countries (the INTERSTROKE study): a case-control study. Lancet 2010;376:112-23.

13. Joshi $P$, Islam $S$, Pais $P$, et al. Risk factors for early myocardial infarction in South Asians compared with individuals in other countries. JAMA 2007;297:286-94.

14. Perumal L, Wells S, Ameratunga S, et al. Markedly different clustering of CVD risk factors in New Zealand Indian and European people but similar risk scores (PREDICT-14). Aust N Z J Public Health 2012;36:141-4.

15. Jenum AK, Diep LM, Holmboe-Ottesen G, et al. Diabetes susceptibility in ethnic minority groups from Turkey, Vietnam, Sri Lanka and Pakistan compared with Norwegians - the association 
with adiposity is strongest for ethnic minority women. BMC Public Health 2012;12:150.

16. Rabanal KS, Lindman AS, Selmer RM, et al. Ethnic differences in risk factors and total risk of cardiovascular disease based on the Norwegian CONOR study. Eur J Prev Cardiol 2013;20:1013-21.

17. Kumar BN, Selmer R, Lindman AS, et al. Ethnic differences in SCORE cardiovascular risk in Oslo, Norway. Eur J Cardiovasc Prev Rehabil 2009;16:229-34.

18. Bannink L, Wells S, Broad J, et al. Web-based assessment of cardiovascular disease risk in routine primary care practice in $\mathrm{New}$ Zealand: the first 18,000 patients (PREDICT CVD-1). N Z Med J 2006;119:U2313

19. Wells S, Riddell T, Kerr A, et al. Cohort Profile: The PREDICT Cardiovascular Disease Cohort in New Zealand Primary Care (PREDICT-CVD 19). Int J Epidemiol 2015:dyv312. dyv312.

20. Statistics New Zealand. Subnational population estimates tables. Wellington: Statistics New Zealand, 2014.

21. New Zealand Guideline Group. The assessment and Management of Cardiovascular Risk. 2003. Wellington, New Zealand.

22. Anderson KM, Odell PM, Wilson PW, et al. Cardiovascular disease risk profiles. Am Heart J 1991;121:293-8.

23. Naess O, Søgaard AJ, Arnesen E, et al. Cohort profile: cohort of Norway (CONOR). Int J Epidemiol 2008:37:481-5.

24. Sulo G, Igland J, Vollset SE, et al. Cardiovascular disease and diabetes mellitus in Norway during 1994-2009 CVDNOR - a nationwide research project. Nor Epidemiol 2013;23.

25. Sulo G, Igland J, Nygård $\mathrm{O}$, et al. Favourable trends in incidence of AMI in Norway during 2001-2009 do not include younger adults: a CVDNOR project. Eur J Prev Cardiol 2014;21:1358-64.

26. Ministry of Health. Wellington. ICD-10-AM/ACH/ACS: Ministry of Health, 2015. Retrieved 25 Sep 2015 from http://www.health.govt.nz/nzhealth-statistics/classification-and-terminology/icd-10-am-achi-acs.

27. Statistics New Zealand. Tatauranga Aotearoa. 2013 Census. Ethnic group profile: Indian. Retrieved 11 Sep 2015 from http://www.stats. govt.nz/Census/2013-census/profile-and-summary-reports/ethnicprofiles.aspx?request_value $=24743 \&$ parent_id $=24726 \&$ tabname $=\#$ 2013.

28. Norwegian Institute of Public Health. Documentation of the CONOR file. Version 02.07.2012. Retrieved 14 Dec 2016 from https://www. fhi.no/globalassets/migrering/dokumenter/pdf/documentation-ofthe-conor-file.pdf.

29. Kumar B, Grøtvedt L, Meyer H, et al. The Oslo immigrant health profile, in Rapport 2008:7. Oslo: Norwegian Institute of Public Health, 2008.

30. Schoenfeld D. Partial residuals for the proportional hazards regression model. Biometrika 1982;69:239-41.

31. Sogaard A. Cohort Norway (CONOR): Materials and methods: Norwegian Institute of Public Health. 2007 http://www.fhi.no/studier/ cohort-of-norway.

32. Tolonen $\mathrm{H}$, Koponen $\mathrm{P}$, Naska A, et al. Challenges in standardization of blood pressure measurement at the population level. BMC Med Res Methodol 2015;15:33.
33. Søgaard AJ, Selmer R, Bjertness E, et al. The Oslo Health Study: The impact of self-selection in a large, population-based survey. Int $J$ Equity Health 2004;3:1-12.

34. Pais P, Pogue J, Gerstein H, et al. Risk factors for acute myocardial infarction in Indians: a case-control study. Lancet 1996;348:358-63.

35. Williams ED, Stamatakis E, Chandola T, et al. Physical activity behaviour and coronary heart disease mortality among South Asian people in the UK: an observational longitudinal study. Heart 2011;97:655-9.

36. Alberti KG, Eckel RH, Grundy SM, et al. Harmonizing the metabolic syndrome: a joint interim statement of the International Diabetes Federation Task Force on Epidemiology and Prevention; National Heart, Lung, and Blood Institute; American Heart Association; World Heart Federation; International Atherosclerosis Society; and International Association for the Study of Obesity. Circulation 2009;120:1640-5.

37. Misra A, Khurana L. The metabolic syndrome in South Asians: epidemiology, determinants, and prevention. Metab Syndr Relat Disord 2009;7:497-514.

38. McKeigue PM, Shah B, Marmot MG. Relation of central obesity and insulin resistance with high diabetes prevalence and cardiovascular risk in South Asians. Lancet 1991;337:382-6.

39. Hjellset VT, Bjørge B, Eriksen HR, et al. Risk factors for type 2 diabetes among female Pakistani immigrants: the InvaDiab-DEPLAN study on Pakistani immigrant women living in Oslo, Norway. J Immigr Minor Health 2011;13:101-10.

40. Ahmed E, El-Menyar A. South Asian ethnicity and cardiovascular risk: the known, the unknown, and the paradox. Angiology 2015;66:405-15.

41. Nair M, Prabhakaran D. Why Do South Asians Have High Risk for CAD? Glob Heart 2012;7:307-14.

42. MacMahon S, Peto R, Cutler J, et al. Blood pressure, stroke, and coronary heart disease. Part 1 , Prolonged differences in blood pressure: prospective observational studies corrected for the regression dilution bias. Lancet 1990;335:765-74.

43. Kumar BN, Meyer HE, Wandel M, et al. Ethnic differences in obesity among immigrants from developing countries, in Oslo, Norway. Int $\mathrm{J}$ Obes 2006;30:684-90.

44. Telle-Hjellset V, Råberg Kjøllesdal MK, Bjørge B, et al. The InnvaDiab-DE-PLAN study: a randomised controlled trial with a culturally adapted education programme improved the risk profile for type 2 diabetes in Pakistani immigrant women. Br J Nutr 2013;109:529-38.

45. Andersen E, Høstmark AT, Holme I, et al. Intervention effects on physical activity and insulin levels in men of Pakistani origin living in Oslo: a randomised controlled trial. J Immigr Minor Health 2013;15:101-10.

46. Rush EC, Chandu V, Plank LD. Reduction of abdominal fat and chronic disease factors by lifestyle change in migrant Asian Indians older than 50 years. Asia Pac J Clin Nutr 2007;16:671-6. 